\title{
An Assessment of School Based Continuous Professional Development (CPD): Practices and Challenges
}

\author{
Bezabih Wondimu \\ Department of Educational Planning and Management, College of Education and Behavioral Studies, Madda Walabu University, Bale-Robe, \\ Ethiopia
}

Email address:

bezabih19@yahoo.com

\section{To cite this article:}

Bezabih Wondimu. An Assessment of School Based Continuous Professional Development (CPD): Practices and Challenges. International Journal of Secondary Education. Vol. 8, No. 1, 2020, pp. 6-15. doi: 10.11648/j.ijsedu.20200801.12

Received: November 11, 2019; Accepted: April 15, 2020; Published: April 29, 2020

\begin{abstract}
The purpose of the study was to assess school based continuous professional development (CPD) with reference to its practices and challenges in secondary schools of Bale Zone, Oromia. Descriptive survey research design was employed. Simple random sampling technique was used to approach individual samples. One hundred thirty six samples, of which (114 teachers, nine principals, nine school CPD facilitators, three districts education supervisors and one zonal education experts) were used for the study. Questionnaire was employed as data gathering tool. Data gathered through questionnaire was analyzed using frequency, percentage, mean and standard deviation while the data gathered through open-ended questions were analyzed qualitatively by means of narration. The findings of the study indicated that practices of school based CPD activities were found inadequate. Lack of common understanding of partners on CPD, absence of link between CPD and teachers' career structure, and absence of giving feedback on CPD activities were identified as major challenges hampering implementation of school based CPD in secondary schools. Prior orientation of teachers and all school partners on overall packages of CPD like induction, mentoring, coaching, action research and portfolio activities; allocating adequate resources used for effective implementation of CPD were forwarded as recommendation of the study.
\end{abstract}

Keywords: Continuous Professional Development, Induction, Mentoring, Coaching, Action Research, Portfolio, Practices and Challenges

\section{Introduction}

\subsection{Background of the Study}

Education is believed to be one of the major forces that speed up economic, social and political advancements in society. It plays a major role in establishing suitable conditions for development process by producing skilled manpower and raising the human capital for national development and it helps to foster changes in technology [1]. Changes in the education system of a nation and global requirements demanded staff development in respective professions. Teachers like most other professional groups, must know the fact that their initial training was not fit throughout the rest of their lives; they need to up-date and upgrade their own knowledge and techniques throughout their lifetime. As a result, there has been an increase in focus on Continuing Professional Development, CPD for teachers worldwide. This is because CPD is continuously viewed as a means of improving learner performance and the production of required skills [2].

Continuous professional development is, therefore, vital for quality education and, teacher development is a never ending cycle of teacher learning that begins with initial teacher training and continuous for as long as a teacher remains in the profession. In support to this viable idea, [3] describes professional development programs as systematic efforts to bring change in the classroom practices of teachers, in their attitudes and beliefs, and in the learning outcome of the students.

Furthermore, the school management bodies, such as principals, department heads, school CPD facilitators, districts and zone education experts are the main motivators in creating shared vision for the curriculum in the school and in providing inspirational curriculum leadership. The instructional activity of leaders determines the success of the 
school and provision of quality education. The school management bodies and school based CPD partners should take the initiative in working together with teachers in designing and implementing developmental programs including the determination of training needs, approaches to satisfy the needs and follow up activities [4].

As education is the key instrument to resolve economic, political, social and cultural problems of a society, there is always a direct interdependence between sustainable development and education. In this respect, Ethiopia has placed education at the center of strategies for development and democratization, with strong policies promoting quality and equity of education [4]. Quality education by itself largely depends on the magnitude of school based teacher's continuous professional development (CPD) in improving learners' achievement. Furthermore, teacher's professional development is a key driver of excellence in any school to contribute to not only teacher and school improvement but also to the overall improvement of education system.

According to [5]. continuous professional development is anything that makes an individual a better teacher targeting at the improvement of teachers' performance in schools. Hence, CPD program is intended to all school teachers, leaders and supervisors in all regions of Ethiopia to participate in high quality and appropriate CPD which positively impacts classroom practices to ensure improved learning. It also allows all teachers to improve their knowledge, skill and attitudes in order that they become more effective classroom practitioners and contribute meaningfully to community development. Moreover, CPD makes quality learning by increasing teachers' skills and knowledge in teaching learning activities [4]. To ensure the quality of education, and improve students result, the professional development of teachers is the most important.

\subsection{Statement of the Problem}

Relevant and Quality Education can be provided for the pupils by involving well qualified teachers at all levels of education (MoE, 2009). Moreover, every education policy places teachers' quality at the very nucleus of learning and as a key determinant of variation in a student achievement. Quality teacher development, however, does not occur by accident. It requires systematic and continuous implementation of teachers' professional enhancement [6]. Thus, teachers are expected to continuously develop and improve their skills, techniques, and knowledge in order to best utilize new curricula and support continuous education reform initiatives. To improve the quality of teachers, the Education and Training Policy (TGE) 1994 set high standards for teachers and described a new approach to education, what we call continuous teachers' professional development (CPD). In the same policy document, much emphasis are given for up grading and updating both in pre- service and in-service teachers to enhance pupils result [4]. [7]. and [8]. also recognized CPD to have a positive impact on the curriculum, improve the classroom activities of teachers and students' academic achievement, pedagogy as well as teacher's effectiveness and their relationship with students. Hence, CPD is very important for teachers to become effective and competent in their Profession in all education levels. Besides, in order to develop the quality of education and improve the professional skill of teachers, the school management bodies have the responsibility of practicing CPD within their schools by arranging workshops, meetings, regular observation of teachers and giving feedback.

However, research studies conducted by different researchers' revealed that implementation of CPD activities are found out to be very poor. For instance, research conducted by [9]. on attitude of teachers towards CPD program, indicated that, teachers have negative attitude towards the use of CPD and hence inadequately implemented in secondary schools of Metekel Zone, Benshangul Gumuz region. Another research conducted by [10]. in overall practices of $\mathrm{CPD}$, in Ethiopian context, and found out that $\mathrm{CPD}$ practices in Ethiopian context was not as indented as mentioned in the policy document.

Hence, to the best knowledge of the writer no study has been conducted on schools based Continuous professional Development, CPD with reference to practices and challenges in some selected secondary schools of Bale Zone, Oromia. In addition none of the research studies has been conducted on the contribution of principals, school CPD facilitators, supervisors and zone educational experts in providing professional support for teachers toward CPD implementation in Bale Zone, Oromia. To this end, to realize the purpose of the study, the following basic research questions were raised and answered in the course of the study.

1. To what extent that school based CPD programs are practiced in secondary schools of Bale Zone Oromia?

2. To what extents that school partners at different levels involved in supporting implementation of school based CPD activities in study areas?

3. What are the challenges hampering effective implementation of school based CPD in secondary schools of Bale zone, Oromia?

\subsection{Conceptual Frameworks of the Study}

Conceptual framework for this study was developed from the premise that theoretical Knowledge is a necessary and important guide for an investigation into school Continuous Professional Development (CPD).

The concept of continuous professional development emanates from the understanding that reflection on selfpractices and collaboration are essential to improve competence and bring quality in education. According to [11]. $\mathrm{CPD}$ is concerned with teaching and learning in school like induction, mentoring, peer coaching, action research, portfolio activities and working with school partnerships. These activities integrate the major practices for successful implementation of CPD program [12].

In order to make the study more manageable and feasible, the study was geographically delimited to secondary schools of Bale Zone, Oromia. Content wise, the study was confound to practices of the CPD programs. Supports given by schools 
partners (principals, school CPD facilitator, supervisors and zone education experts) were the domains of the study. Challenges hampering effective implementation of CPD program was also considered as a focus areas of the study. Therefore, in this study, Variables such as CPD activities (induction, mentoring, peer coaching, action research, and portfolio activities) would be treated as an independent variable and their effect on improvement of teacher's professional development would be treated as dependent variable.

\subsection{Research Methods and Design}

\subsubsection{Research Method}

Research methods are styles of conducting a research work which are determined by the nature of the problem [13]. To this effect, in this study, quantitative research approach more specifically, a descriptive cross-sectional survey design was employed. Hence, writer of this article used the descriptive survey research design for two main reasons: firstly, descriptive design helps the researcher to obtain current information concerning the practices and challenges of CPD in secondary schools. A descriptive survey, according to [14], is a method of collecting information by administering a questionnaire to a sample of individuals. This design was appropriate for this study because the writer collected data from secondary school teachers, principals, school CPD facilitators, supervisors, districts and zone education office experts in order to investigate the practices and challenges of CPD.

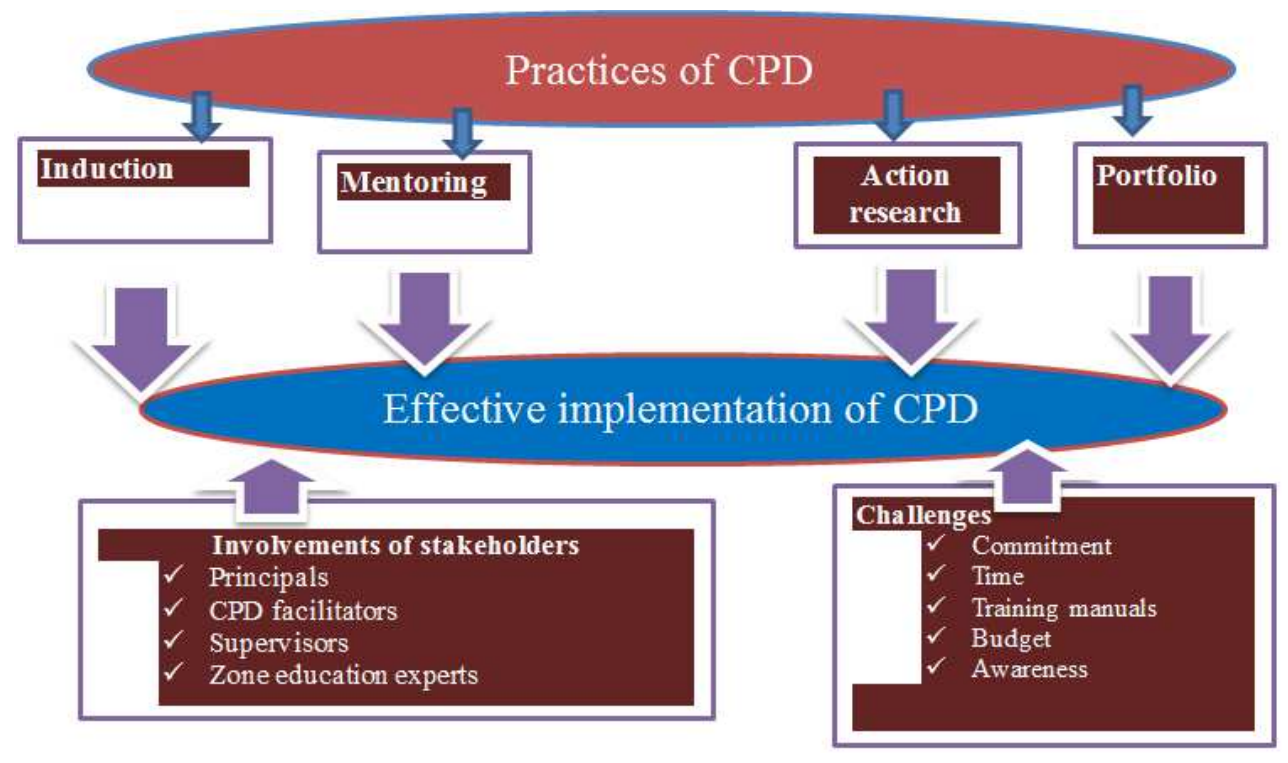

Figure 1. Conceptual framework of the study [15].

\subsubsection{Sources of Data}

Primary sources of data were used in the study. Primary sources of data were collected from secondary school teachers, principals, supervisors, schools CPD facilitators, districts and zone education experts. The very reasons for selecting these as primary sources of data with the assumption that they have a direct relation with and also have rich information about the practice of CPD program in schools and the challenges they encounter.

\subsubsection{Study Area, Population, Sample Size and Sampling Technique}

The study area for this research was secondary schools of Bale Zone, Oromia. Of 22 districts in Bale zone, 9 districts (namely Goba, Sinana, Dello Mena, Ginnir, Jara, Gassera, Agarfa, Dinisho, and Robe town) were randomly selected as a subject of the study. In line with this, one secondary schools from each districts was randomly selected as samples of the study. In areas under study, there are 560 teachers, 66 principals, 22 school CPD facilitators, 5 supervisors and one zone education experts working in coordinating CPD in schools. From this all total population of the study, 136 samples, of which 114 teachers, 9 principals, 9 school CPD facilitators, 3 districts educational supervisors and one zonal educational experts) were randomly selected and used for the study.

\subsubsection{Data Collection Instruments}

In this study, questionnaire was employed as an appropriate data collection tool to collect relevant data from selected secondary school teachers, principals, school CPD facilitators, districts and zone education experts. The data provided by questionnaires can be more easily analyzed and interpreted than the data obtained from verbal responses. Questionnaires were in written forms that asked exact questions of all individuals in the sample group, and which respondents can answer at their own convenience [15]. The prepared questionnaires were spread for the samples of the study and the result was statistically computed by the SPSS 21 program. The questionnaires were framed in likert scale type with five options (Very high=5, High=4, Medium=3, Low $=2$ and Very Low $=1$ ); and it consists both close ended and open ended questions.

\subsubsection{Checking for Validity and Reliability of Instruments}


According to [16]. validity refers to the degree to which an instrument measures what it is supposed to measure. To be sure of the validity of instruments, initially the instrument was prepared and commented by experts in the field. Based on their comments, the instruments were improved before they were administered to the major participants of the study to reduce errors. To check the reliability, the questionnaires were pilot tested to check the truthfulness of the instruments with the objective of the study. Hissu Secondary School teachers were taken through simple random sampling technique to fill the questionnaires. Then, the prepared questionnaires were spread for the sample teachers for the pilot study. Therefore, the 21 teachers, 1 principal and 1 school CPD facilitator of Hissu secondary school filled the questionnaires. The result of the pilot testing was statistically computed by the SPSS 21 program. The Cronbach's Alpha Model was used for analysis of data. Based on the pilot test, the reliability coefficient of the instrument was found to be $0.85(85 \%)$ and, hence, was taken to be reliable. Thus, the instrument was found to be reliable as statistical literature recommend a test result of 0.75 (75\% reliability) and above as reliable [17]. The table below indicates the computed reliability coefficient of the pilot study.

Table 1. Reliability coefficient of the practices and challenges of school based CPD.

\begin{tabular}{llll}
\hline No & Major categories of practices and challenges of CPD & No of items & Reliability coefficient \\
\hline 1 & School based CPD practices & 5 & 0.82 \\
2 & Teachers implementation of the activities of CPD & 5 & 0.88 \\
3 & The Support of School based CPD partners & 20 & 0.84 \\
4 & The challenges of CPD implementation & 6 & 0.85 \\
Average & & 0.85 \\
\hline
\end{tabular}

\subsubsection{Methods of Data Analysis}

Quantitative data analysis technique was employed. The data collected through closed ended questionnaire was tallied, tabulated and filled in to SPSS version 21 and analysis was made with the help of frequency, percentage, mean and standard deviation. Likert scale was employed to identify to what extent the respondents replied very high to very low. The rating consists of five scales; $5=$ very high, 4=high, $3=$ medium, $2=$ low and $1=$ very low. For ease analysis and interpretation, the writer used 3.0 as expected mean. The interpretations be made using norm reference for all five point scale measurements based on the following mean score results. $1.00-1.49=$ very low, $1.50-2.49=$ low, $2.50-$ $3.49=$ medium, $3.50-4.49=$ high, $4.50-5.00=$ very high [18]. The data gathered through open-ended questions were analyzed qualitatively through descriptive narration for the purpose of triangulation. Finally, the findings were summarized and suggested recommendations were forwarded.

\section{Data Presentation, Analysis and Interpretation}

The overall purpose of the study was to assess school based continuous professional development (CPD) with references to its practices and challenges in some selected secondary schools of Bale Zone, Oromia. To realize purpose of the study, a total of 136 questionnaires, 114 for teachers, 9 principals, 9 school CPD facilitators, 3 supervisors and 1 zone education experts were distributed and data analyses were done based on the data obtained from the questionnaires.

\subsection{The Practices of CPD Implementation}

This part deals with the items related to the implementation of CPD practices by secondary schools teachers. Each item was analyzed based on the data obtained through questionnaires responded by 114 teachers, 9 principals, 9 school CPD facilitators, 3 supervisors and 1 zone education experts and further checked backed by open ended questions presented to the respondents. Therefore, the five items were analyzed interpreted as indicated in the table below.

As indicated in item 1 of table 2, the respondents were asked to answer about the extent in which their involvement on action research. Accordingly, 16 (12\%) and 21 (16.5\%) of them replied that, high and medium respectively. On the other hand, $99(80 \%)$ of them replied as low. The calculated mean values was found out to be $(\mathrm{m}=2.38$ with $\mathrm{SD}=1.1)$ which is within the scope of low level. However, the findings contrary to the literature, the practice of action research to solve the teaching learning activities at school was ineffective [5].

Table 2. Practices of CPD implementation.

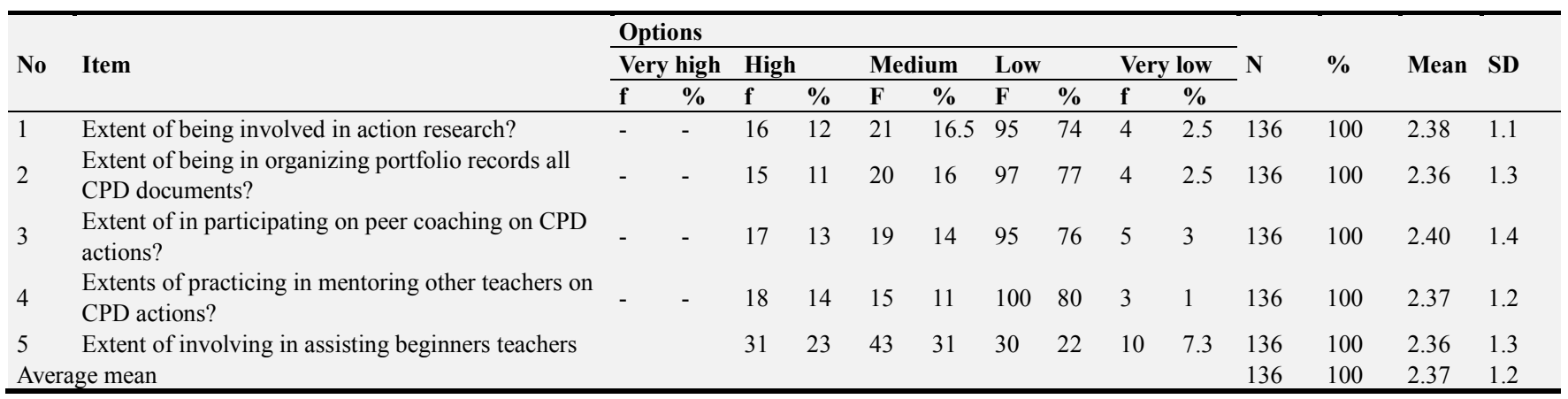


In item 2 of table 2, the respondents were asked to rate about their answer to extent in which the action of organizing portfolio was compiled complete record of all CPD documents. Accordingly, $15(11 \%)$ and $20(16 \%)$ of them rated that high and medium respectively. $101(82 \%)$ of them responded as low. The calculated mean values was found out to be $(\mathrm{m}=2.38$ with $\mathrm{SD}=1.3)$ which is in the range of low level. From the data, it can be said that teachers' preparation of CPD portfolio by recording all relevant documents was found to be insufficient. By the document analysis it was confirmed that teachers did not prepare CPD portfolio by recording all useful actions of CPD.

In support to the response to the questionnaire, majority of the respondents responded to open ended question as read as follows:

Majority of teachers have portfolio. However, their portfolios were similar. This can be a reason that, many teacher copy from each other. The other was that feedback was not given on portfolio but there were signs showing that the portfolio was done, the activities most mentioned in the portfolio are continuous assessment, applying active learning and doing action research and there is lack of information about the format and the purpose of the portfolio at all levels. Because of that there is no uniformity. Teachers prepare portfolio but not well compiled, don't included all the activities in the manual.

In item 3 of table 3, respondents were asked to answer regarding the extent to which participating on peer coaching on CPD actions. Accordingly, 17 (13\%) and 19 (15\%) of them responded that, high and medium respectively. On the other hand, $100(82 \%)$ of them rated as low. The calculated mean values was found out to be $(\mathrm{m}=2.40$ with $\mathrm{SD}=1.4)$ which is in the range of low level. This indicated that the degree to which participation of teachers on peer coaching on CPD action was low.

In item 4 of table 4 , the respondents were asked to answer about the extent to which practicing to mentoring other teachers on CPD action. Accordingly, 18 (14\%) and 15 (11\%) of them replied as high and medium respectively. On the other hand, $103(83 \%)$ of them replied as low. The calculated mean values was found out to be $(\mathrm{m}=2.37$ with its $\mathrm{SD}=1.2$.) which is in the scope of low level. This indicated that the mentoring activities of experienced teachers for newly deployed teachers were not practically implemented. This showed that the mentoring activities were not accomplished at the expected level.

In item 5 of the same table, the respondents were asked to answer about the extent to which induction training was given to beginner teachers. Accordingly, 22 (16\%) and 31 $(23 \%)$ of them replied high and medium respectively. On the contrary, $43(31 \%)$ of them responded low. The calculated mean values was found out to be $(\mathrm{m}=2.3$ with its $\mathrm{SD}=1.3$ ) this implied that induction training was rarely given beginner teachers in secondary schools of Bale zone, Oromia.

The overall calculated average mean value for five items were calculated and found out to be $(m=2.37$, with its $\mathrm{SD}=1.2$ ) indicating that the degree of implementation of CPD practices was found to be low.

In support to the response to the questionnaire, majority of the respondents responded to open ended question as read as follows:

All school CPD partner (principals, CPD facilitators, supervisors, districts and zone educational experts) confirmed that the practices of CPD activities were found out to be low in all schools under study.

\subsection{Contributions of CPD stakeholders}

\subsubsection{The Contribution of Schools Principals on CPD Practices}

Teachers' CPD is an ongoing and systematic and supported by the school principals. The principal, therefore, is responsible for helping teachers to grow and develop in their understanding of teaching and class room life in improving basic teaching skills and in expanding their knowledge. This part of analysis examined whether principals effectively support teachers professional development activities in order to help teachers develop in their profession.

Table 3. The support of school principals in enhancing CPD.

\begin{tabular}{|c|c|c|c|c|c|c|c|c|c|c|c|c|c|c|c|}
\hline \multirow{3}{*}{ No } & \multirow{3}{*}{ Item } & \multicolumn{10}{|c|}{ Options } & \multirow{3}{*}{$\mathbf{N}$} & \multirow{3}{*}{$\%$} & \multirow{3}{*}{ Mean } & \multirow{3}{*}{ SD } \\
\hline & & \multicolumn{2}{|c|}{ Very high } & \multicolumn{2}{|c|}{ High } & \multicolumn{2}{|c|}{ Medium } & \multicolumn{2}{|c|}{ Low } & \multicolumn{2}{|c|}{ Very low } & & & & \\
\hline & & $\mathbf{F}$ & $\%$ & f & $\%$ & f & $\%$ & f & $\%$ & $\mathbf{F}$ & $\%$ & & & & \\
\hline 1 & $\begin{array}{l}\text { Principals' effort to prepare training on CPD } \\
\text { activities for teachers? }\end{array}$ & - & - & 16 & 12 & 20 & 15 & 90 & 65 & 10 & 6 & 136 & 100 & 2.38 & 1.1 \\
\hline 2 & $\begin{array}{l}\text { Principals effort to organize experience } \\
\text { sharing among the Nearby schools? }\end{array}$ & - & - & 14 & 10 & 21 & 16 & 89 & 64 & 12 & 4 & 136 & 100 & 2.37 & 1.3 \\
\hline 3 & $\begin{array}{l}\text { Principals' effort to produce annual CPD } \\
\text { plans with teachers? }\end{array}$ & - & - & 15 & 11 & 20 & 15 & 90 & 65 & 11 & 3 & 136 & 100 & 2.36 & 1.2 \\
\hline 4 & $\begin{array}{l}\text { Principals' effort to organize induction } \\
\text { programs for newly deployed teachers? }\end{array}$ & - & - & 17 & 12.5 & 21 & 16 & 88 & 63 & 10 & 6 & 136 & 100 & 2.40 & 1.3 \\
\hline 5 & $\begin{array}{l}\text { Principals' effort to evaluate the CPD } \\
\text { activities of teachers? }\end{array}$ & - & - & 17 & 12.5 & 15 & 11 & 94 & 66 & 10 & 6 & 136 & 100 & 2.35 & 1.1 \\
\hline 6 & $\begin{array}{l}\text { Principal's effort to give feedback on the } \\
\text { actions of CPD? }\end{array}$ & - & - & 15 & 11 & 20 & 15 & 88 & 63 & 13 & 6.5 & 136 & 100 & 2.36 & 1.2 \\
\hline \multicolumn{2}{|r|}{ Average mean } & & & & & & & & & & & 136 & 100 & 2.37 & 1.2 \\
\hline
\end{tabular}

In item 1 of table 3 , the respondents were asked to rate 
on CPD activities for teachers. Accordingly, 16 (12\%) and 20 $(15 \%)$ of them replied that, high and medium respectively. Moreover, $100(73 \%)$ and $(\mathrm{m}=2.38$ with its $\mathrm{SD}=1.1)$ which showed that, principals effort to prepare training on $\mathrm{CPD}$ activities for teachers were low level.

In item 2 of table 3 , the respondents were asked to rate about the extent to which principals' effort to organize experience sharing among the nearby schools. Accordingly, $14(10 \%)$ and $21(16 \%)$ of them replied that, high and medium respectively. On the other hand, 100 (74\%) were responded as low. The calculated mean value for item was found out to be ( $\mathrm{m}=2.37$ with its $\mathrm{SD}=1.3$ ) this indicates that the roles of principals' effort to arrange experience sharing among the nearby schools were low.

In support to the response to the questionnaire, majority of the respondents responded to open ended question as read as follows:

Principals do not encourage creativity and innovation, not understand CPD well, not trained well, and does not provide teachers with the necessary support. In addition, Districts and zone education experts confirmed that the efforts of school principals to organize experience sharing, ensuring the quality of involvement of teachers in CPD activities, and giving constructive feedback were not effectively implemented.

In item 3 of the same table, the respondents were asked to rate about the extent to which principals' effort to produce annual CPD plan with teachers. Accordingly, 15 (11\%) and $20(14 \%)$ were responded that, high and medium respectively. On the other hand, 101 (75\%) replied as low. The calculated mean value for the item was found out to be $(\mathrm{m}=2.36$ with its $\mathrm{SD}=1.3$ ) which are in the scope of low level of participation. This implied that the effort of principals to produce annual CPD plan with teachers' participation was low at the school level.

In item 4 of the above table the respondents were asked to rate about the extent to which principals' effort to organize induction programs for newly deployed teachers. Accordingly, 17 (13\%) and 21 (16\%) of them responded high and medium respectively. On the contrary, 98 (71\%) of them responded as low. The calculated mean value for the item was found out to be $(\mathrm{m}=2.40$ with its $\mathrm{SD}=1.3)$ which are in the scope of low level of support. This implied that principal's effort to arrange induction programs for newly deployed teachers and to develop self-confidence and to avoid unnecessary tension and future malfunction were low.

Regarding item 5 of table 3, respondents were asked to rate the extent to which principals' effort to evaluate the CPD activities of teachers. Accordingly, 17 (13\%) and 15 (11\%) of them replied high and medium respectively. In addition, 104 (76\%) of them responded as low. The calculated mean value for the item was found out to be $(\mathrm{m}=2.35$ with its $\mathrm{SD}=1.1)$ which is inclined in the low level of evaluating and giving feedback on CPD activities of teachers by principals.

Item 6 of table 3, teachers were asked to rate the extent to which, principals effort to give feedback on the actions of CPD. Accordingly, 15 (11\%) and 20 (15\%) of them replied high and medium respectively. On the other hand, 101 (74\%) of them replied as low. The calculated mean value for the item was found out to be $(\mathrm{m}=2.36$ with its $\mathrm{SD}=1.2)$ which showed low level of support from school principals to enhancing the practices of CPD.

The overall calculated average mean value for five items were calculated and found out to be $(m=2.37$, with its $\mathrm{SD}=1.2$ ) indicating low level of support from school principals to enhancing the practices of CPD.

In support to the response to the questionnaire, majority of the respondents responded to open ended question as read as follows:

School based CPD facilitators and cluster supervisors replied that principals do not fully visit the activities of CPD and the general actions of teaching and learning and to discuss with students to solve the problems. This implied that the monitoring and evaluation of CPD activities were not effective enough at school level and also, from the results it can be said that continuous evaluation of the success of CPD activities was low.

From this, it is possible to conclude that, most of the principals paid attention to routine administrative activities rather than CPD activities. This implies that principals give less attention to CPD activities. In contrary to this idea, scholars have indicated that the implementation of CPD activities requires good school managing and favorable school environment [5]. Therefore, principals, teachers, education experts and other concerned bodies should attempt to improve the program through a joint effort in order to achieve the objectives of CPD. Otherwise, the vision to produce professionally well informed and motivated teachers, capable of delivering quality education will not be fulfilled.

\subsubsection{The Contribution of CPD Facilitators Enhancing the Practices of CPD.}

This title was treated with the aim of assessing the level of effectiveness of the secondary schools CPD facilitators in encouraging school based CPD.

Table 4. The support of school CPD facilitators in enhancing CPD practices.

\begin{tabular}{|c|c|c|c|c|c|c|c|c|c|c|c|c|c|c|c|}
\hline \multirow{3}{*}{ No } & \multirow{3}{*}{ Item } & \multicolumn{10}{|c|}{ Options } & \multirow{3}{*}{$\mathbf{N}$} & \multirow{3}{*}{$\%$} & \multirow{3}{*}{ Mean } & \multirow{3}{*}{ SD } \\
\hline & & \multicolumn{2}{|c|}{ Very high } & \multicolumn{2}{|c|}{ High } & \multicolumn{2}{|c|}{ Medium } & \multicolumn{2}{|l|}{ Low } & \multicolumn{2}{|c|}{ Very low } & & & & \\
\hline & & f & $\%$ & f & $\%$ & f & $\%$ & $\mathbf{F}$ & $\%$ & $\mathbf{F}$ & $\%$ & & & & \\
\hline 1 & $\begin{array}{l}\text { CPD facilitators arrange discussion with } \\
\text { senior teachers? }\end{array}$ & - & - & 18 & 13 & 15 & 11 & 100 & 75 & 3 & 1 & 136 & 100 & 2.37 & 1.2 \\
\hline 2 & $\begin{array}{l}\text { Effort of CPD facilitators give training on } \\
\text { CPD practices according to teachers need? }\end{array}$ & - & - & 15 & 23 & 20 & 31 & 97 & 22 & 4 & 7.3 & 136 & 100 & 2.36 & 1.3 \\
\hline 3 & $\begin{array}{l}\text { Effort of CPD facilitators to arrange colleague } \\
\text { evaluation on the implementation of CPD? }\end{array}$ & - & - & 17 & 24 & 21 & 29 & 95 & 23 & 4 & 9 & 136 & 100 & 2.38 & 1.2 \\
\hline \multicolumn{2}{|c|}{ Average mean } & & & & & & & & & & & 136 & 100 & 2.37 & 1.2 \\
\hline
\end{tabular}


In item 1 of table 4 , the respondents were asked to rate about the extent to which CPD facilitators arrange discussion with senior teachers. Accordingly, 18 (13\%) and 15 (11\%) of them replied that high and medium respectively. On the other hand, $103(76 \%)$ of them replied as low. The calculated mean value for item was found out to be $(\mathrm{m}=2.37$ with its $\mathrm{SD}=1.2)$ within the range of low level of support at school level. This showed that CPD facilitators inadequately prepare discussion with senior teachers within the school on CPD practices.

In item 2 of the same table, the respondents were asked to rate about the extent to which effort of CPD facilitators to give training on CPD practices according to teachers need. Accordingly, 15 (11\%) and $20(15 \%)$ of them replied that, high and medium respectively. On the other hand, 101 (74\%) of them replied as low. The calculated mean value for item was found out to be $(m=2.36$ with its $\mathrm{SD}=1.2)$ which are in the scope of low level of support. This indicated that the support of CPD facilitators to give training on CPD activities was inadequate.

Item 3 of table 4 , teachers were asked to rate about the extent to which the efforts of CPD facilitators to arrange colleague evaluation on the implementation of CPD. Accordingly, $17(13 \%)$ and $21(16 \%)$ of them replied that high and medium respectively. $99(71 \%)$ of them responded as low. As we can see from the above table, the calculated mean value for item was found out to be $(m=2.38$ with its $\mathrm{SD}=1.3$ ) which are in the scope of low level of support. This implied that in order to organize colleague evaluation on CPD action, the effort of CPD facilitators was insufficient.

In line with closed ended questionnaires, respondents express their views to open ended question follows:

$C P D$ facilitators have lack of knowledge about CPD, were less commitment to provide technical assistance for teachers, have not good awareness about CPD. This it is possible to conclude that school CPD facilitators were give less support on the activities of CPD.

The overall calculated average mean value for five items were calculated and found out to be $(m=2.37$, with its $\mathrm{SD}=1.2$ ) which are in the range of low level of support and not practiced as the expected point.

In contrary, CPD facilitators arrange discussion with senior teachers. Peer support and discussion with senior teachers can contribute towards the development and take -up of new practices, and further discussion progression [19].

\subsubsection{The Contribution of Supervisor and Districts Education Experts on CPD Implementation}

This part of the study displays the items with regard to the degree of support from cluster supervisors and districts education experts in the process of implementing CPD activities.

Table 5. Support of cluster supervisors and districts educational experts.

\begin{tabular}{|c|c|c|c|c|c|c|c|c|c|c|c|c|c|c|c|}
\hline \multirow{3}{*}{ No } & \multirow{3}{*}{ Item } & \multicolumn{10}{|c|}{ Options } & \multirow{3}{*}{$\mathbf{N}$} & \multirow{3}{*}{$\%$} & \multirow{3}{*}{ Mean } & \multirow{3}{*}{ SD } \\
\hline & & \multicolumn{2}{|c|}{ Very $\mathbf{H}$} & \multicolumn{2}{|c|}{ High } & \multicolumn{2}{|c|}{ Medium } & \multicolumn{2}{|c|}{ Low } & \multicolumn{2}{|c|}{ Very low } & & & & \\
\hline & & f & $\%$ & f & $\%$ & f & $\%$ & f & $\%$ & $\mathbf{F}$ & $\%$ & & & & \\
\hline 1 & $\begin{array}{l}\text { Effort of supervisors and wereda educational } \\
\text { experts follow up to school CPD }\end{array}$ & - & - & 18 & 14 & 15 & 11 & 100 & 74 & 3 & 1 & 136 & 100 & 2.37 & 1.2 \\
\hline 2 & $\begin{array}{l}\text { Effort of maintain experience sharing among the } \\
\text { cluster schools? }\end{array}$ & - & - & 17 & 13 & 19 & 14 & 95 & 71 & 5 & 3 & 136 & 100 & 2.38 & 1.3 \\
\hline 3 & Extent to arranging training regarding CPD? & - & - & 15 & 11 & 20 & 16 & 90 & 65 & 11 & 5 & 136 & 100 & 2.37 & 1.2 \\
\hline \multicolumn{2}{|r|}{ Average mean } & & & & & & & & & & & 136 & 100 & 2.37 & 1.2 \\
\hline
\end{tabular}

In item 1 of table 5 , the respondents were asked to rate about the extent to which cluster supervisors and districts educational experts effort to follow up the school CPD performance. Accordingly, 18 (14) and 15 (11\%) of them replied that, high and medium respectively. On the contrary, 103 (75) of them responded as low. The calculated mean value for item was found out to be $(\mathrm{m}=2.37$ with its $\mathrm{SD}=1.2)$ which align towards low. From the data, it could be learned that cluster supervisors effort to follow up the school CPD performance was found to be at the low level of support.

In item 2 of table 5 , the respondents were asked to rate about the need to see the efforts of cluster supervisors and districts education experts to maintain experience sharing among the cluster school. Accordingly, 17 (13\%) and 19 (14\%) of them responded that, high and medium respectively. On the contrary, $100(73 \%)$ of the responded as low. The calculated mean value for item was found out to be $(m=2.38$ with its $\mathrm{SD}=1.3$ ) which are in the scope of low level of support. This showed that the effort of cluster supervisors to coordinate experience sharing among the cluster schools was much unsatisfactory. Continuous professional development (CPD) can be effective if and only if cluster supervisors are knowledgeable, committed, willing, qualified and experienced.

In item 3 of table 5 , the respondents were asked to rate about the extent to which the supports of school based CPD supervisors and Districts education office experts to arrange training regarding CPD actions. Accordingly, 15 (11\%) and $20(15 \%)$ of them replied that, high and medium respectively. However, 100 (74\%) of them responded as low. The calculated mean value for item was found out to be $(\mathrm{m}=2.37$ with its $\mathrm{SD}=1.2$ ) which are in the range of low level. This indicated that Districts education office has given less attention to prepared training on the actions of CPD.

Item 4 of table 5 , teachers were asked to rate about the extent to which the efforts of districts education office experts to give feedback on the activities of CPD. Accordingly, 14 (9\%) and $21(17 \%)$ of them responded that, high and medium respectively. On the other hand, 101 (69\%) of them replied as low. The calculated mean value for item 
was found out to be $(\mathrm{m}=2.37$ with its $\mathrm{SD}=1.2)$ which are in the scope of low level.

The overall calculated average mean value for five items were calculated and found out to be $(\mathrm{m}=2.37$, with its $\mathrm{SD}=1.2)$ implying that implied that, the support of supervisors and districts education office experts to give feedback on the actions of CPD was unsatisfactory at school level.

The response of the open-ended questionnaire also confirmed that:

CPD practice is not effectively implemented because of less support of supervisors in the area. Most of them focus on political issues and engagement in routine activities. This implies that they gave less attention to their role in the implementation of CPD.

Un-coinciding with the findings of this study, Vanniekerk (2002), indicated that the implementation of CPD activities requires good school managing and favorable school environment Vanniekerk (2002). In support to this idea, CPD guideline of MoE (2009), in the education system the districts education offices play an important role in the implementation of CPD program. Districts Education Offices (WEOs) are responsible to producing local CPD plans, ensuring that all schools have annual CPD plans, monitoring and evaluating the CPD activities of schools, collecting data about CPD activities in the districts, collecting data of individuals' and schools' participation in CPD.

\subsubsection{Contribution of Zone Education Office Experts on CPD Implementation}

This part of the study displays the items with regard to the degree of support from zone education experts in the process of implementing CPD activities. Whatever attempts were made at the various levels, it is meaningless unless supervisory services or activities are provided for schools.

Table 6. Support provided by zone education office experts to implement CPD.

\begin{tabular}{|c|c|c|c|c|c|c|c|c|c|c|c|c|c|c|c|}
\hline \multirow{3}{*}{ No } & \multirow{3}{*}{ Item } & \multicolumn{10}{|c|}{ Options } & \multirow{3}{*}{$\mathbf{N}$} & \multirow{3}{*}{$\%$} & \multirow{3}{*}{ Mean } & \multirow{3}{*}{ SD } \\
\hline & & \multicolumn{2}{|c|}{ Very high } & \multicolumn{2}{|c|}{ High } & \multicolumn{2}{|c|}{ Medium } & \multicolumn{2}{|c|}{ Low } & \multicolumn{2}{|c|}{ Very low } & & & & \\
\hline & & f & $\%$ & f & $\%$ & $\mathbf{F}$ & $\%$ & $\mathbf{F}$ & $\%$ & f & $\%$ & & & & \\
\hline 1 & $\begin{array}{l}\text { Extent Zone education office experts deliver training } \\
\text { for teachers to implement CPD actions? }\end{array}$ & - & - & 17 & 13 & 21 & 16 & 95 & 69 & 4 & 2 & 136 & 100 & 2.38 & 1.3 \\
\hline 2 & $\begin{array}{l}\text { Extent Zone education office experts support to } \\
\text { allocate resources needed to implement CPD activities? }\end{array}$ & - & - & 18 & 14 & 15 & 11 & 100 & 72 & 6 & 3 & 136 & 100 & 2.37 & 1.2 \\
\hline 3 & $\begin{array}{l}\text { Extent the support of Zone education office experts } \\
\text { effectively monitoring CPD activities? }\end{array}$ & - & - & 15 & 11 & 20 & 16 & 97 & 61 & 16 & 12 & 136 & 100 & 2.36 & 1.1 \\
\hline \multicolumn{2}{|r|}{ Average mean } & & & & & & & & & & & 136 & 100 & 2.37 & 1.2 \\
\hline
\end{tabular}

In item 1, of table 6 , the respondents were asked to rate about the extent to which zone education office experts' support to deliver training for teachers to implement CPD actions. Accordingly, 17 (13\%) and 21 (16\%) of them replied that, high and medium respectively. On the other hand, 99 $(71 \%)$ of them replied as low. The calculated mean value for item was found out to be $(\mathrm{m}=2.38$ with its $\mathrm{SD}=1.3)$ which are in the range of low level of support. Thus, it can be said that zone education office experts' support to deliver training for teachers to implement the CPD action was insufficient in each secondary schools in Bale zone, Oromia.

In item 2 of table 6 , teachers were asked to give their responses about the extent to which zone education office experts allocate resources needed to implement the CPD activities. Accordingly, $18(14 \%)$ and $15(11 \%)$ of them replied that, high and medium respectively. On the other hand $103(75 \%)$ of them responded as low. The calculated mean value for item was found out to be $(m=2.37$ with its $\mathrm{SD}=1.2$ ) which are in the range of low. This indicated that zone education office expert's support on CPD actions were low level. Thus, it could be concluded that zone education office experts has given some support for CPD activities.

In item 3 , of table 6 , the respondents were asked to rate about the extent to which the supports of zone education office experts to monitor and evaluate the CPD activities. Accordingly, 15 (11\%) and 20 (17\%) of them rated that, high and medium respectively. On the other hand, 101 (72\%) of them replied as low. The calculated mean value for item was found out to be $(m=2.37$ with its $\mathrm{SD}=1.1)$ which are in the scope of low level of support.

The overall calculated average mean value for three items were calculated and found out to be $(\mathrm{m}=2.37$, with its $\mathrm{SD}=1.2$ ) which are in the range of low level of support. This indicated that continuous monitoring and evaluation system of CPD in zone education office experts on the activities of CPD was low in the secondary schools of Bale zoe, Oromia.

In the same way, the response to open ended questionnaires also revealed that the supervisory assistance provided by zone experts was not regular and frequent. All the participants agreed that the zone experts' visit at secondary schools with a maximum of twice in a semester one can say that, zone and districts education office experts support for secondary schools were ineffective in activating and facilitating the actual implementation of school based CPD.

In addition, supporting this ideas, majority of the respondents' responded to an open ended question and expressed their views as follows:

Most of the time, Zone and District Education office use to visit schools only when there is a problem. There is no culture of dialogue and there is limited professional support. There is also no timely feedback on the important issues of $C P D$. Further they responded as they were not play an important role in monitoring, evaluating and training in the activities of CPD at school level due to shortage of budget.

In contrary, zone education departments have various responsibilities such as; analyzing and identifying regional priorities, production of materials and delivering training to 
implement them, sharing information with all stakeholders, annually producing and circulating regional CPD, allocating the resources needed to implement the regional CPD program including the development of Teachers' Professional Portfolios [1].

\subsection{The Challenges of School Based CPD}

This section is devoted to the presentation of the major difficulties that hinder the positive application of CPD activities. In line with this, open ended questions were presented to teachers, principals, school CPD facilitators, supervisors and zone education experts; and all of them replied in similar ways to open ended questions. For instance one of the school principle expressed his views as read as follows:

The main obstacles that operate against the practice and Challenges of school based CPD were less committeemen of school leaders to implement CPD activities, shortage of time for teachers to implement the CPD actions, lack of trained CPD facilitators, lack of teachers motivation on the actions of CPD, lack of arranging training on CPD programs, lack of resources or budget to implement $\mathrm{CPD}$, absence of committeemen of teachers to implement the CPD program, lack of awareness of teachers on CPD activities, the absence of career structure with the actions of CPD was found to be the major challenges hampering effective implementation of school based CPD in study areas.

Coinciding with this study, ministry of education, MOE (2010b), the major challenges identified at the national level are lack of trained facilitators, high turnovers of more experienced and trained leaders or facilitators and stakeholders extra work load, particularly of teachers. Teachers are not motivated by the districts to alleviate the ongoing problems. CPD books are not sufficiently prepared by the languages of work. Teachers are not provided awareness about the background of CPD. Teachers and other responsible partners are not well oriented how to implement CPD in collaboration with other pillars of quality education. Thus, the school based CPD program is not being realized in collaboration with other education quality improvement programs.

\section{Major Findings, Conclusion, and Recommendation}

This section presents the description of the major findings, recommendations forwarded based on the data

\subsection{Summary of Major Findings}

On the basis of the analysis and interpretation of the data, the following major findings were made.

1. The practices of school based Continuous professional development program for teachers should aim at forming a better and more effective teacher capable of adapting to different school or classroom situations. However, the findings of the study indicated that teacher's well oriented how to implement CPD priority areas (induction, mentoring, coaching, action research and portfolios) and were nor oriented how to implement CPD activities in collaboration with other CPD partners.

2. As indicated in the study, teachers' participation in CPD priority areas induction, mentoring, coaching, action research and portfolios) and collaborative work with CPD partners were found out to be insufficient. This implies that there was inadequate teachers' involvement in the activities of CPD.

3. Among the responsibilities expected from schools principals are:-preparing training or workshops, organize experience sharing with the nearby schools, arrange induction program for newly deployed teachers, evaluating and giving feedback on CPD activities of teachers. Therefore, from the data of the study, it is possible to conclude that teachers were not encouraged by the school principals to accomplish the school based CPD activities and develop their professional skills in order to bring into the light them with low trends in their respective subjects.

4. Districts and zone education office experts, cluster supervisors and other school based CPD implementers were low supportive on the activities of CPD. That is, there was failure to organize induction program for newly deployed teachers, failure to allocate sufficient budget, absence of preparing local CPD plans, organizing training programs, intra- and inter group discussions, encouraging peer evaluation and timely feedback regarding the practical implementation of school based CPD. Therefore, there was absence of committeemen and lack of responsibilities among CPD stakeholders for its real implementation.

5. It was revealed that principals made less effort in collaborating with WEB and ZEB professionals to ensure the national CPD priorities whereas principal's effort to organize induction program, experience sharing and monitor and assess the contents of individual professional portfolios and giving constructive feedback was at medium level. From this, it is possible to conclude that teachers were not encouraged by the school principals to accomplish the CPD activities and develop their professional skills.

6. The result of this study showed that the main obstacles that operate against the practice and Challenges of school based CPD were less committeemen of school leaders to implement CPD activities, shortage of time for teachers to implement the CPD actions, lack of trained CPD facilitators, lack of teachers motivation on the actions of CPD, lack of arranging training on CPD programs, lack of resources or budget to implement CPD, absence of commitment of teachers to implement the CPD program, lack of awareness of teachers on CPD activities, the absence of career structure with the actions of CPD was found to be the major challenges hampering effective implementation of school based CPD in study areas. 
In sum, it is possible to conclude that school based continuous professional development was inadequately implemented so that ambition to attain quality education was below the expected in secondary schools of Bale zone, Oromia

\subsection{Recommendations}

Based on the major findings of the study, the following recommendations were forwarded.

1. CPD practices (induction, mentoring coaching action research and portfolio activities) are found out to be low. Hence, MoE, Regional education Bureau, zone education department, and districts education offices are advised to consider to implement school based CPD as part of the performance of teachers' career structure development criteria and it needs immediate actions in order to solve the problem.

2. The contributions of schools principals to CPD program implementation were not sufficient. Hence, it is recommended that, the schools principals must committed to school based CPD implementation and to assist teachers, preparing workshops, organize experience sharing with the nearby schools, arrange induction program for newly deployed teachers, evaluating and giving feedback on CPD activities to teachers in developing and achieving educational objectives in line with the needs of schools.

3. Support of school CPD facilitators were found out to be insignificant in all secondary schools in Bale zone, Oromia. Hence, it is recommended that facilitators should practically assist teachers by facilitating conducive environment for skill development through both short and long term training programs, by preparing self and peer evaluation opportunities and followed by giving timely feedback for teachers.

4. The study results showed that support from cluster supervisors and districts education experts for the implementation of school based CPD were found to be inadequate. Hence, it is recommended that cluster supervisors should take immediate actions and follow up the school CPD performance, organize teachers training on the activities of CPD and maintain experience sharing with the nearby cluster schools and share CPD manuals and materials to all secondary schools for the real implementation of school based CPD.

\section{References}

[1] MoE (1994). New education and training policy. Addis Ababa: EMPDA. Directions, London: Kogan page ltd.
[2] Coolahon J (2002). Teacher education and the Teaching career in on education working Paper, Number, 2. Education Directorate, OECD, Paris.

[3] Guskey $\mathrm{T}$ and sparks D (2002). Linking professional development to improvements in student learning, Paper presented at the American Educational Research Association, New Orleans, LA.

[4] Sparks D (2002). a New Vision for staff Development. Paper co-published by the National Staff Development council (NSDC) and the Association for Supervision and Curriculum development (ASCD).

[5] MoE (2009). Teachers, leaders and supervisors in Ethiopia framework, Ministry of Education. Ethiopia. MoE (2009). CPD for primary and secondary school Teachers, Leaders and supervisors in

[6] TGE (1994). New education and training policy. Addis Ababa: EMPDA. USAIDAED/BESO II project (2006). Supervision, Leadership, Community Mobilization: A. A.

[7] Fraser C, Kennedy A, Reid L and Mckinney S (2005). Teachers continuing Professional Development, contested concepts, understandings and Models Professional Development in Education, 33 (2) 153- 169

[8] Fullon M (2006). Leading a culture of change. San Francisco: Jossey - Bass.

[9] Hargreaves A (1994). Changing teachers changing times: Teachers work and culture in the postmodern age, London: Cassel.

[10] Yitayew (2013). Practice and challenges of cpd in primary schools of metekel zone, Jimma University. Ethiopia unpublished MA thesis.

[11] Jonanson B (1991). Teacher as researcher. Taking action research to task, USA.

[12] David G (2006). Professional Development for Leaders and management of self-Governing Schools Newcastle, the Netherlands and Austria: Springer Printing Press.

[13] Orodho AJ (2003). Essentials of Educational and Social Sciences Research Method. Nairobi: Masola Publishers.

[14] Gall T, Gall P and Borg W (2007). Educational research. An introduction (8th Ed.) Toronto, on: Ally\&Bacon.

[15] George D and Malley P (2003). Calculating, interpreting, and reporting Cronbach's Alpha Reliability Coefficient for Likert Scales. Middle West Research to Practice Conference in Adult, continuing, and Community Education, 87-88.

[16] Lee L (2002). The effect of Agency Training for Talwanese child care director Professional Development (china), (Electronic resource). Ed. D. Thesis at spaldine University.

[17] MoE (2010). Continuous professional Development for primary and secondary school

[18] Bell T, et-al. (2001). Teacher Development a model from science education, London: the Flamer Press. 